\title{
Charmed-hadron production and charm fragmentation at ZEUS
}

\author{
William Dunne
}

University of Glasgow

on behalf of the ZEUS collaboration

\author{
April 17, 2006
}




\section{Introduction}

Previous measurements of charm production

Aims and Motivations of new measurements

Charm fragmentation

Definition

Hadron Reconstruction

Fractions

Overview

Variables

Disentangling $D^{* \prime} \mathrm{s}$

$R_{u / d}$

Vector Mesons

$P_{V}^{d}$

$\mathrm{S}$ suppression

Summary \& Outlook 


\section{Previous inclusive $D^{*}$ DIS measurement}

- Inclusive $c$

\section{ZEUS}

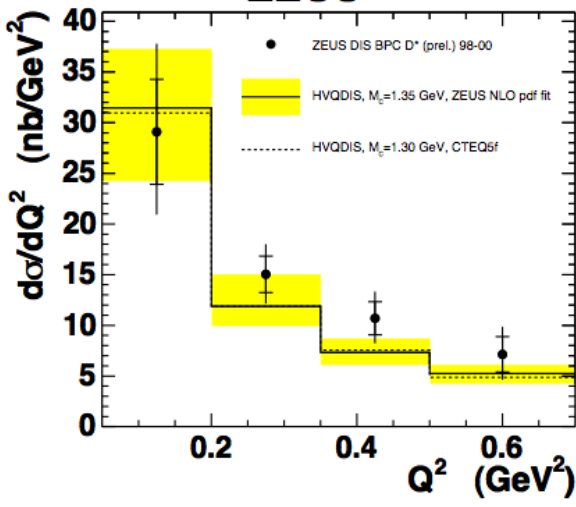

cross-sections have been measured previously at ZEUS

ZEUS-prel-04-032

- $82 \mathrm{pb}^{-1}$

- $p_{T}\left(D^{*}\right)<9 \mathrm{GeV}$, $\left|\eta\left(D^{*}\right)\right|<1.6$

- $0.05<Q^{2}<0.7$

- probe transition region

- Differential $\sigma\left(D^{*}\right)$

Shape well described by NLO 


\section{Aims and Motivations}

- Follow Eur. Phys. J. C. 44, 351-366 (2005)

- Reconstruct the charm mesons $D^{0}, D^{ \pm}, D^{* \pm}, D_{s}^{ \pm}$and the charm baryon $\Lambda_{c}^{ \pm}$. Hereafter $D^{+}, \Lambda^{+}$is taken to mean $D^{ \pm}, \Lambda^{ \pm}$

- Use measurements of the cross sections of those hadrons to obtain

- Fragmentation fractions

$\rightarrow$ Are fragmentation fractions independent of experiment?

- Strange suppression

$\rightarrow$ How frequently are s quarks picked up by c quarks in $D$ mesons?

- Ratio of $u / d$ production

$\rightarrow$ Are $u$ and $d$ quark picked up equally by c quarks in $D$ mesons?

- Integrated luminosity of $79 \mathrm{pb}^{-1}$ in photoproduction regime 


\section{Charm fragmentation fractions}

The fraction of $c$ quarks hadronising as a particular charm hadron is given by

$$
f\left(c \rightarrow D, \Lambda_{c}\right)=\frac{\sigma_{D, \wedge c}}{\text { Total } \sigma_{g s}}
$$

- $\sigma_{D, \wedge c}$ - production cross section $(\sigma)$ for the hadron

- Total $\sigma_{g s}$ - sum of production $\sigma$ for all $c$ ground states (ie not $D^{*+}$ ) that decay weakly

- Dominated by: $D^{+}, D^{0}, D_{s}^{+}$and $\Lambda_{c}^{+}$

- Charm-strange baryons $\Xi_{c}^{+}, \Xi_{c}^{0}$ and $\Omega_{c}^{0}$ included by estimating they contribute $14 \%$ of $\Lambda_{c}^{+}$

The $\sigma_{D^{+}}$and $\sigma_{D^{0}}$ contributions to Total $\sigma_{g s}$ are the sums of their direct cross section and $D^{*}$ decay contribution

$$
\text { By definition } \Sigma_{g s} f\left(c \rightarrow D, \Lambda_{c}\right)=1
$$




\section{Reconstruction of charmed hadrons}

- $D^{*+}, D^{+}, D^{0}, D_{s}^{+}$, and $\Lambda_{c}^{+}$are all measured in PHP in the same kinematic range $p_{T}\left(D, \Lambda_{C}\right)>3.8 \mathrm{GeV}$ OR $\left|\eta\left(D, \wedge_{c}\right)\right|<1.6$

- Background reduction gained by cutting on $p_{T}$ and decay angles of the decay products

- Problem: Some $D^{*+}$ decay into $D^{0}$ 's which fall outside of the kinematic range

- We must keep track of those $D^{0}$ 's which arise from $D^{*+}$ decay to avoid double counting in $\sigma\left(D^{*+}\right)$

- Solution: We divide up the $D^{0}$ sample into those that originate from a $D^{*+}$ decay and those that do not 


\section{Separation of $D^{0}$ sample}

- For $D^{0}$ candidates a search is made for a soft pion in a $D^{*+} \rightarrow D^{0} \pi^{+}$decay and tagged if this is the case

- $D^{*+} \rightarrow D^{0}+\pi_{s}$ events are considered a sum of two subsamples

- 'Tagged' events with $p_{T}\left(D^{0}\right)>3.8 \mathrm{GeV}$ and $\left|\eta\left(D^{0}\right)\right|<1.6$

- 'Additional' events with $p_{T}\left(D^{0}\right)<3.8 \mathrm{GeV}$ and $\left|\eta\left(D^{0}\right)\right|>1.6$

- The 1st sample is represented by labeling these $D^{0}$ events with a special 'tag'.

- The 2nd sample is a set of separately measured 'additional' $D^{*}$ events

- $\sigma^{\mathrm{kin}}\left(D^{*+}\right)=\sigma^{\mathrm{add}}\left(D^{*+}\right)+\sigma^{\operatorname{tag}}\left(D^{0}\right) / B_{D^{*} \rightarrow D^{0} \pi^{+}}$ 


\section{Subtraction of Reflections}

- We must ensure in the mass region about the signal there is no contribution from any other decay modes

- This would provide a false signal and must be corrected for

- List decays with same number of daughter particles in this mass region

- Obtain reflections by assigning masses to tracks which correspond to these daughter particles

- These reflection shapes taken from MC and normalised by signals in nominal decay modes

- Reflections are much wider than signal and provide complex background shapes

- Signal fits are more stable after reflection subtraction 


\section{The Modified Gaussian}

- Mass distributions were fitted with a 'modified' Gaussian function + background

$$
\text { Gauss }^{\text {mod }} \propto \exp \left[-0.5 x^{1+1 /(1+0.5 x)}\right]
$$

- $x=\left(\left[M-M_{D}\right] / \sigma\right)$

- Background function is:

- linear for $\Lambda_{c}^{+}$and $D^{+}$

- exponential for $D_{s}^{+}$and $D^{0}$

- polynomial for $D^{*+}$

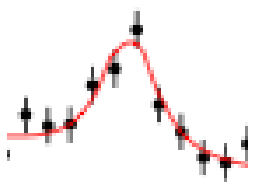

- Modified Gaussian has 3 free parameters like the regular Gaussian $\rightarrow$ far superior fit to data and MC signals $\rightarrow$ especially useful for high statistics MC signals 


\section{Reconstruction of $\Lambda_{c}^{+}$baryons}

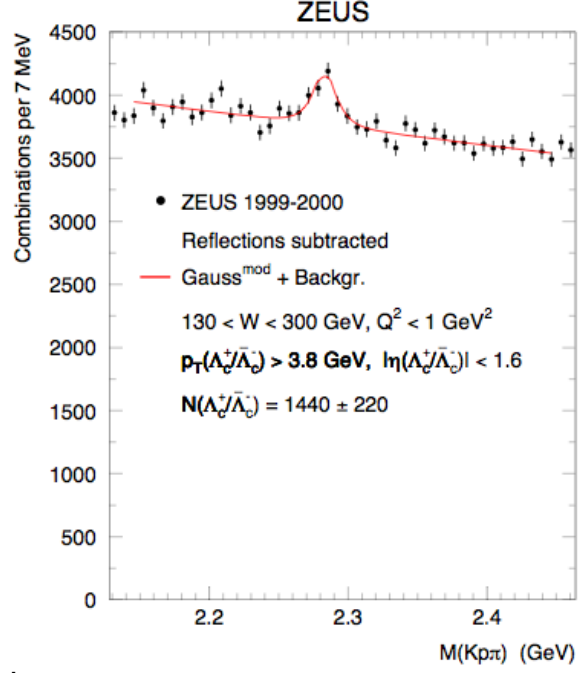

- $M(K p \pi)$ spectrum after all cuts

- $d E / d x$ cuts applied to suppress background

- Reflections from $D^{+}$ $D_{s}^{+}$to 3 charged particles subtracted using the simulated reflection shapes. 


\section{Reconstruction of additional $D^{*+}$ mesons}

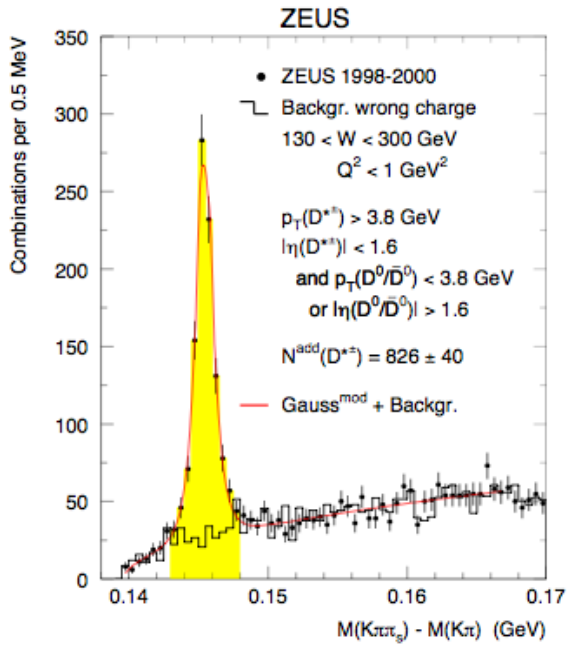

- $\triangle M$ spectrum

- $N\left(D^{*+}\right)$ counted by subtracting wrong charge background in yellow region

- $D^{* \prime}$ s counted in range:

$0.143<\Delta M<0.148$ $\mathrm{GeV}$ 


\section{Reconstruction of $D_{s}^{+}$mesons}

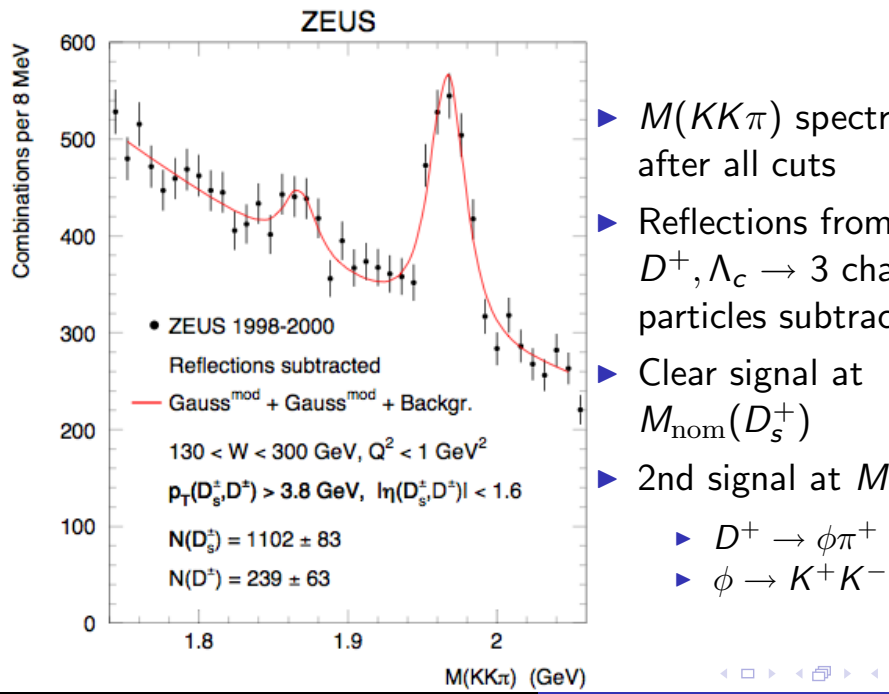




\section{Reconstruction of $D^{0}$ mesons}

\section{ZEUS}

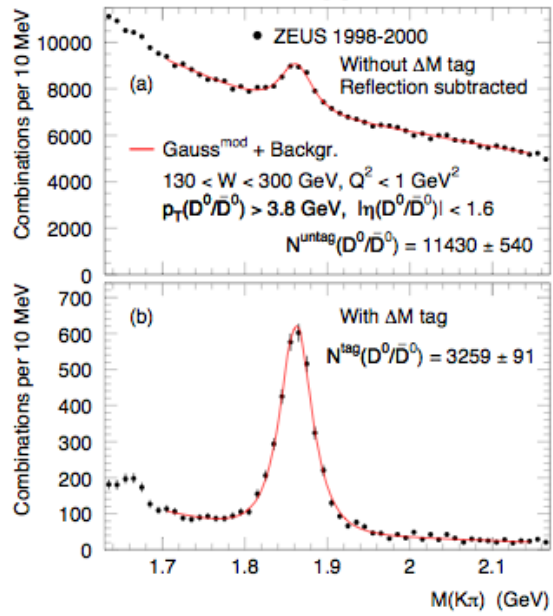

- $D^{0}$ 's are tagged if they originate from a $D^{*+}$ decay

- Later necessary to remove the $D^{*+}$ contribution to $D^{0}$

- Tagged $D^{0}$ signal is more pronounced

- Fit performed simultaneously 


\section{Reconstruction of $D^{+}$mesons}

\section{ZEUS}

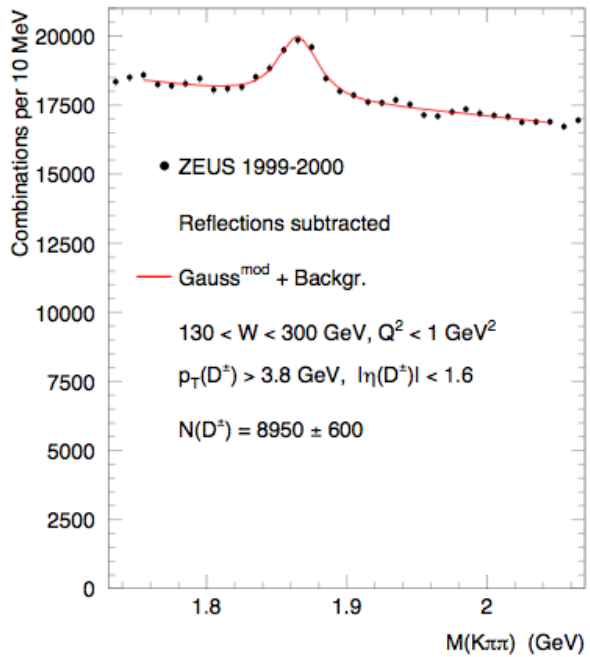

- Reflections from $D_{s}^{+}, \Lambda_{c} \rightarrow 3$ charged particles subtracted

- All signals are resolved cleanly at ZEUS 


\section{Fragmentation fractions for $\Lambda_{c}^{+}$}

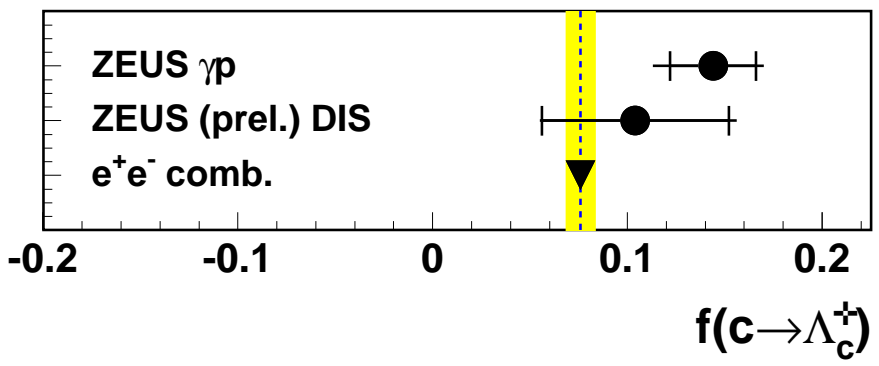

- Decay channel: $\Lambda_{c}^{+} \rightarrow K^{-} p \pi^{+}$

- Larger than but consistent with world average 


\section{Fragmentation fractions for $D^{*}$}

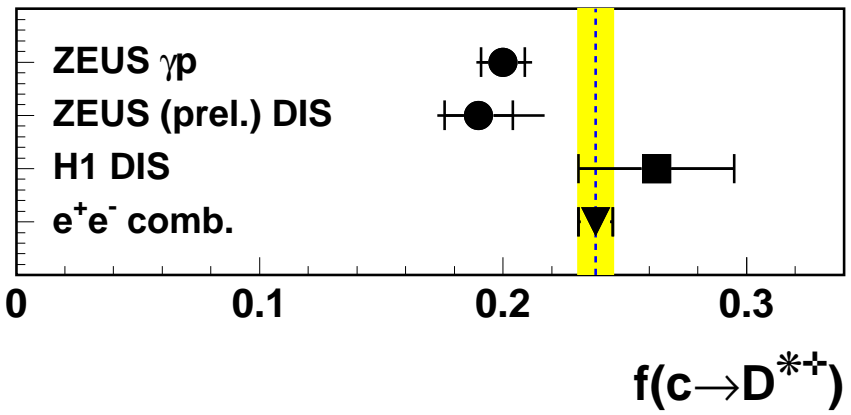

- Decay channel: $D^{*+} \rightarrow D^{0} \pi_{s}^{+}$

- Smaller than but consistent with previous measurements

- About half of the difference due to low $f\left(c \rightarrow \Lambda_{c}^{+}\right)$ 


\section{Fragmentation fractions for $D_{s}^{+}$}

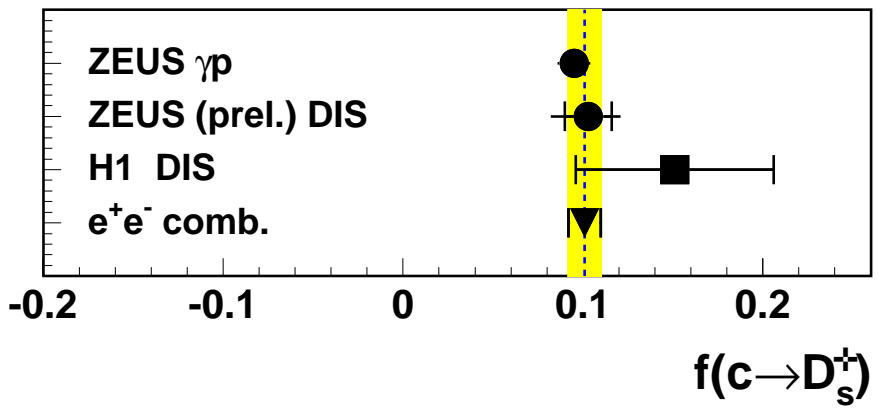

- Decay channel: $D_{s}^{+} \rightarrow \phi^{0}\left(\rightarrow K^{+} K^{-}\right) \pi^{+}$

- Excellent agreement 


\section{Fragmentation fractions for $D^{0}$}

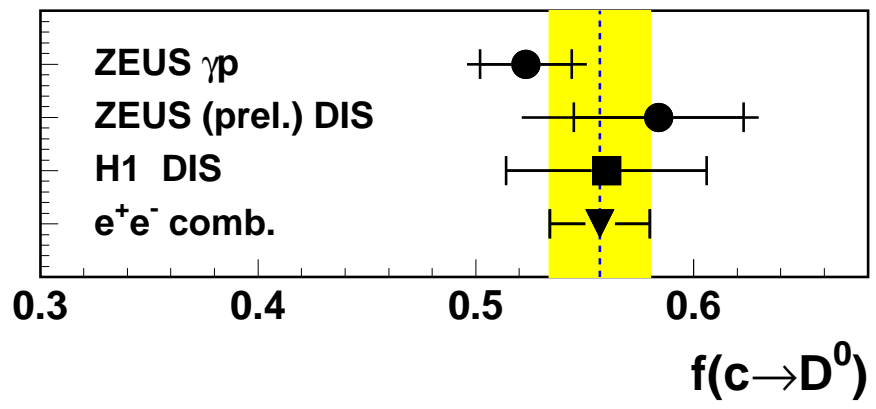

- Decay channel: $D^{0} \rightarrow K^{-} \pi^{+}$

- Good agreement 


\section{Fragmentation fractions for $D^{+}$}

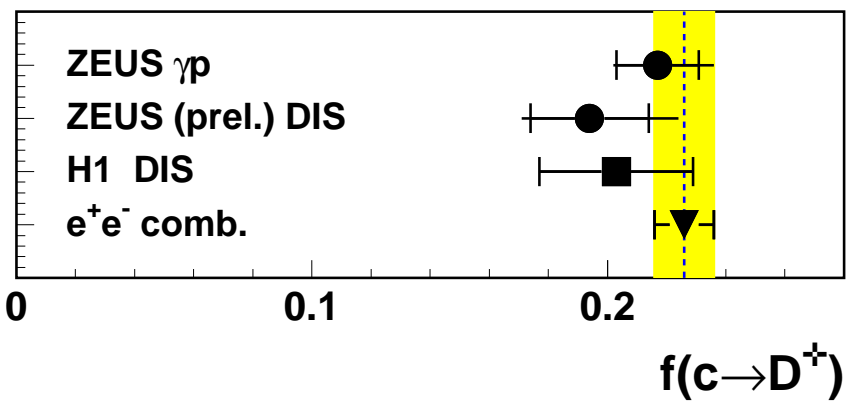

- Decay channel: $D^{+} \rightarrow K^{-} \pi^{+} \pi^{+}$

- Excellent agreement 


\section{Overview: Charm fragmentation fractions}

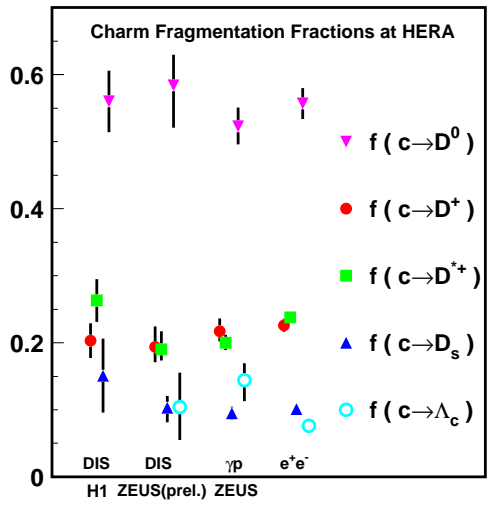

Consistent with universality assumption of charm fragmentation 


\section{Disentangling $D^{* \prime s}$}

"In the measurement of $f(c \rightarrow X)$ it is sometimes necessary to disentangle which of the decay modes have a contribution from a $D^{*}$ decay and which do not. Often it is necessary to subtract this contribution before making a measurement."

- Recall $D^{0}$ 's are tagged if they are a result of a $D^{*+}$ decay

- Any $D^{*+}$ which give rise to a $D^{0}$ outwith the kinematic cuts are called additional $D^{*+}$

- The measured quantities in this analysis are

- $\sigma^{\text {untag }}\left(D^{0}\right)$ : The production cross section for $D^{0}$ mesons not originating from the $D^{*+} \rightarrow D^{0} \pi_{s}^{+}$decay

- $\sigma^{\operatorname{tag}}\left(D^{0}\right)$ : The production cross section for $D^{0}$ mesons originating from the $D^{*+} \rightarrow D^{0} \pi_{s}^{+}$decay

- $\sigma^{\text {add }}\left(D^{*+}\right)$ : The production cross section for additional $D^{*+}$ mesons $\left(p_{T}\left(D^{0}\right)<3.8 \mathrm{GeV}\right.$ OR $\left.\eta\left(D^{0}\right)>1.6\right)$

- give list of equations? like sigma kin relationships etc 


\section{$R_{u / d}$ : Relative number of $u$ and $d$ quarks}

The ratio of neutral to charged $D$ meson production rates

$$
R_{u / d}=\frac{\sigma^{\operatorname{dir}}\left(D^{0}\right)+\sigma\left(D^{* 0}\right)}{\sigma^{\operatorname{dir}}\left(D^{+}\right)+\sigma\left(D^{*+}\right)}
$$

- We are now dealing with both excited and ground state mesons. Care must be taken to treat $D^{*}$ contribution correctly

- $\sigma^{\operatorname{dir}}\left(D^{0}\right)$ and $\sigma^{\operatorname{dir}}\left(D^{+}\right)$are those parts of $\sigma\left(D^{0}\right)$ and $\sigma\left(D^{+}\right)$ not originating from $D^{*}$ decays

- Problem: $\sigma^{\operatorname{dir}}\left(D^{0}\right)$ and $\sigma^{\operatorname{dir}}\left(D^{+}\right)$are not measured 


\section{$R_{u / d}$ : Relative number of $u$ and $d$ quarks}

- To express this formula in terms of quantities measured at ZEUS we make use of a number of relationships

- Since $D^{* 0} \rightarrow D^{0}+X$ always

$$
\text { - } \sigma^{\operatorname{dir}}\left(D^{0}\right)+\sigma\left(D^{* 0}\right)=\sigma^{\text {untag }}\left(D^{0}\right) \text { (i.e. not from } D^{*+} \text { ) }
$$

- $\sigma^{\operatorname{dir}}\left(D^{+}\right)=\sigma\left(D^{+}\right)$- the contribution from $D^{*+}$

$$
\text { - } \left.\sigma^{\operatorname{dir}}\left(D^{+}\right)=\sigma\left(D^{+}\right)-\sigma\left(D^{*+}\right) \dot{(1}-B_{D^{*+} \rightarrow D_{\pi^{+}}^{0}}\right)
$$

- By substitution we arrive at the usable expression

$$
R_{u / d}=\frac{\sigma^{\mathrm{untag}}\left(D^{0}\right)}{\sigma^{\operatorname{tag}}\left(D^{0}\right)+\sigma\left(D^{+}\right)}
$$




\section{$R_{u / d}$ : Relative number of $u$ and $d$ quarks}

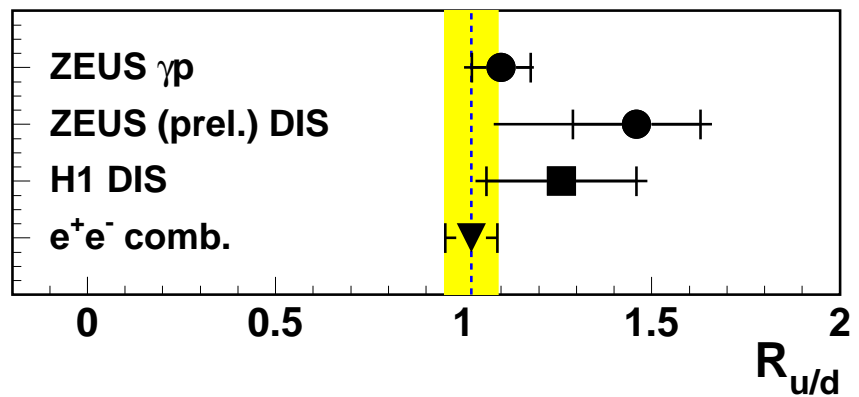

$$
R_{u / d}=\frac{\sigma^{\operatorname{dir}}\left(D^{0}\right)+\sigma\left(D^{* 0}\right)}{\sigma^{\operatorname{dir}}\left(D^{+}\right)+\sigma\left(D^{*+}\right)}=\frac{c \bar{u}}{c \bar{d}}
$$

- $u$ and $d$ quarks are produced equally in charm fragmentation $\rightarrow$ Strong Isospin Invariance Holds 


\section{Fraction of Vector Meson Production}

$3 \mathrm{VM}$ spin states 1 PS spin state

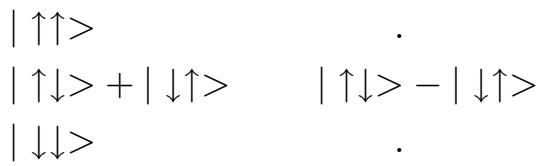

- The number of permitted spin states:

- Vector mesons $\left(D^{*+}\right)$ have a total of 3

- Pseudoscalar mesons $\left(D^{0}, D^{+}\right)$have only 1 .

- By naive spin counting we expect $D$ mesons to be produced in a VM state $3 \times$ more often than in a PS state - $\frac{v}{V+P S}=0.75$

- Can be verified by measurements of $D^{*+}$ and $D^{+}$ 


\section{Fraction of Vector Meson Production}

The fraction of $D$ mesons produced in a vector state

$$
P_{V}^{d}=\frac{V}{V+P S}=\frac{\sigma^{\operatorname{kin}}\left(D^{*+}\right)}{\sigma^{\operatorname{kin}}\left(D^{*+}\right)+\sigma^{\operatorname{dir}}\left(D^{+}\right)}
$$

- $\sigma^{\text {kin }}\left(D^{*+}\right)=$ the production $\sigma\left(D^{*+}\right)$ in an equivalent kinematic range to $\sigma^{\mathrm{dir}}\left(D^{+}\right)$

$$
p_{T}\left(D^{*}\right)>3.8 \mathrm{GeV},\left|\eta\left(D^{*}\right)\right|<1.6
$$

- $\sigma^{\operatorname{dir}}\left(D^{+}\right)=$the part of $\sigma\left(D^{+}\right)$not from $D^{*+}$ decays

$$
P_{V}^{d}=\frac{\sigma^{\operatorname{tag}}\left(D^{0}\right) / B_{D^{*+} \rightarrow D^{0} \pi^{+}}+\sigma^{\text {add }}\left(D^{*+}\right)}{\sigma\left(D^{+}\right)+\sigma^{\operatorname{tag}}\left(D^{0}\right)+\sigma^{\text {add }}\left(D^{*+}\right)}
$$




\section{$P_{V}^{d}$ : Fraction of $\mathrm{VM}$ in $\mathrm{f}(c \rightarrow D)$ fragmentation}

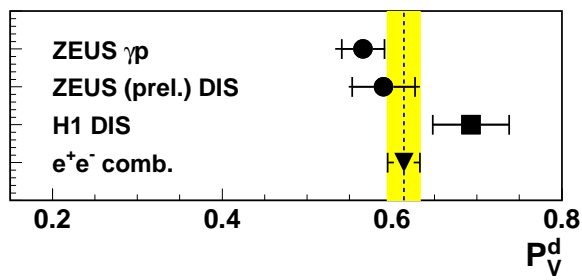

- ZEUS: $P_{V}^{d}=0.566 \pm 0.025$ (stat) ${ }_{-0.022}^{+0.007}$ (syst. $)_{-0.023}^{+0.022}$ (br)

- Smaller than but consistent with previous measurements

- Considerably smaller than simple spin counting prediction.

- Thermodynamical \& String Fragm. models: $P_{V}^{d} \approx 0.666$

- closer to but still above the measured value

- $P_{V}^{D} \neq 0.75$. Simple spin counting does NOT work with charm 


\section{Strangeness suppression factor}

"Strangeness suppression is a parameter which determines the ratio of probabilities to create a $s$ to $u, d$ quark in the fragmentation process."

$$
\gamma_{s}=\frac{2 \sigma\left(D_{s}^{+}\right)}{\sigma^{\mathrm{eq}}\left(D^{+}\right)+\sigma^{\mathrm{eq}}\left(D^{0}\right)}
$$

- Using relationships for the equivalent cross sections $\sigma^{\mathrm{eq}}(D)$

$$
\begin{gathered}
\sigma^{\mathrm{eq}}\left(D^{0}\right)=\sigma^{\mathrm{untag}}\left(D^{0}\right)+\sigma^{\operatorname{tag}}\left(D^{0}\right)+\sigma^{\mathrm{add}}\left(D^{*+}\right)\left(R_{u / d}+B_{D^{*+} \rightarrow D^{0} \pi^{+}}\right) \\
\sigma^{\mathrm{eq}}\left(D^{+}\right)=\sigma\left(D^{+}\right)+\sigma^{\mathrm{add}}\left(D^{*+}\right)\left(1-B_{D^{*+} \rightarrow D^{0} \pi^{+}}\right) \\
\gamma_{s}=\frac{2 \sigma\left(D_{s}^{+}\right)}{\sigma\left(D^{+}\right)+\sigma^{\operatorname{untag}}\left(D^{0}\right)+\sigma^{\operatorname{tag}}\left(D^{0}\right)+\sigma^{\operatorname{add}}\left(D^{*+}\right)\left(1+R_{u / d}\right)}
\end{gathered}
$$




\section{Strangeness suppression factor}

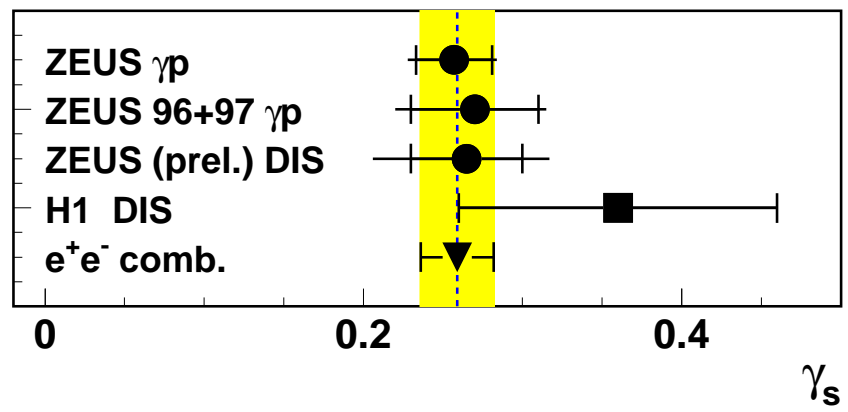

- The strangeness suppression factor in $c$ fragmentation $\approx \frac{1}{3.9}$ 


\section{Summary \& Outlook}

- Summary

- Fragmentation fractions are consistent with universality

- Ratio of $u$ to $d$ is consistent with Isospin invariance

- Vector to Pseudoscalar ratio has been measured is inconsistent with naive spin counting

- HERA II outlook

- More luminosity

- MVD will reconstruct secondary vertices and measure lower $P_{t}$ 


\section{BONUS SLIDES}

There now follows a selection of additional slides for the interested reader 


\section{Reconstruction $\Lambda_{c}^{+}$baryons}

- Decay channel: $\Lambda_{c}^{+} \rightarrow K^{-} p \pi^{+}$

- $\Lambda_{c}^{+}$constructed from tracks of ++- charge configuration

- Given that $M(p)>>M(\pi)$

- $M(p)$ is assigned to the + track with highest $p$

- Remaining + track is assigned $M(\pi)$

- $M(K)$ assigned to remaining track and $M(K p \pi)$ is calculated

- To reduce background:

- $p_{T}(K)>0.75, p_{T}(p)>1.3, p_{T}(\pi)>0.5 \mathrm{GeV}$

- $\cos \theta^{*}(K)<-0.9$.

- $\cos \theta^{*}(p)>0.25$.

- $p^{*}(\pi)>90 \mathrm{MeV} \cdot p^{*}(\pi)$ is $p(\pi)$ in the $\Lambda_{c}^{+}$rest frame

- Cuts on $d E / d x$ values of decay tracks

$\theta^{*}(X)$ is $\theta$ between $X$ in $\Lambda_{c}^{+}$rest frame and $\Lambda_{c}^{+}$line of flight in lab 
$D^{*+}$

\section{Reconstruction of additional $D^{*+}$ mesons}

- Decay channel: $D^{*+} \rightarrow D^{0}\left(\rightarrow K^{-} \pi^{+}\right) \pi_{s}^{+}$

- $D^{*+}$ constructed from tracks of -++ configuration

-+- tracks with $p_{T}>0.4 \mathrm{GeV}$ combined in pairs to form $D^{0}$ candidates

- $K$ and $\pi$ masses assumed in turn and $M(K \pi)$ calculated

- $D^{0}$ candidate kept provided

- $1.81<M(K \pi)<1.92 \mathrm{GeV}$

- and either $p_{T}\left(D^{0}\right)<3.8 \mathrm{GeV}$ or $|\eta|>1.6$

- Remaining + track assigned $M(\pi)$ if $p_{T}>0.2 \mathrm{GeV}$

- $\Delta M=M\left(D^{*+}\right)-M\left(D^{0}\right)$ histogrammed 
$D_{s}^{+}$

\section{Reconstruction of $D_{s}^{+}$mesons}

- Decay channel: $D_{s}^{+} \rightarrow \phi\left(\rightarrow K^{+} K^{-}\right) \pi^{+}$

- Oppositely charged tracks with $p_{T}>0.7 \mathrm{GeV}$ assigned $M(K)$ to form $\phi$ candidates

- $\phi$ candidate kept if $M(\phi)-8 \mathrm{MeV}<M(K K)<M(\phi)+8 \mathrm{MeV}$

- Any additional $p_{T}>0.5 \mathrm{GeV}$ track combined with $\phi$ to form $D_{s}^{+}$candidate

- to reduce background:

- $\cos \theta^{*}<0.85$. $\theta^{*}$ is angle between $\pi$ in $D^{*+}$ rest frame and $D^{*+}$ line of flight in lab frame

- $\left|\cos ^{3} \theta^{\prime}(K)\right|>0.1$. theta' $(K)$ is angle between $K$ and $\pi$ in $\phi$ rest frame. Motivated by spin alignments. 


\section{Reconstruction of $D^{0}$ mesons}

- Decay channel: $D^{0} \rightarrow K^{-} \pi^{+}$

- Oppositely charged tracks with $p_{T}>0.8 \mathrm{GeV}$ from $D^{0}$ candidates

- $K$ and $\pi$ masses are assigned to each track $\rightarrow M(K \pi)$ calculated

- Rest frame angle between $K, \pi \theta^{*}(K)$ is cut $\left|\cos \theta^{*}(K)\right|<0.88$

- Ambiguity in $K, \pi$ assignment to tracks is corrected for by 'tagging' $D^{0}$ 's arising from $D^{*+} \rightarrow D^{0} \pi_{s}$

- In this decay $K, \pi$ are correctly assigned to $D^{0}$ decay tracks

- This $D^{0}$ spectrum using incorrect assignment is normalised by $N^{\text {untag }}\left(D^{0}\right) / N^{\text {tagged }}\left(D^{0}\right)$ and subtracted from the untagged $D^{0}$ spectrum. 


\section{Reconstruction of $D^{+}$mesons}

- Decay channel: $D^{+} \rightarrow K^{-} \pi^{+} \pi^{+}$

- Like charged tracks with $p_{T}>0.5 \mathrm{GeV}$ combine with third opposite charge track to from $D^{+}$candidates

- $\pi$ masses are assigned to both like charged tracks, $K$ mass to third and $M(K \pi \pi)$ calculated

- Angle between $K$ in $D^{+}$rest frame and $D^{+}$line of flight in lab frame is cut at $\left|\cos \theta^{*}(K)\right|<0.88$

- Background reduced by removing $M(K \pi \pi)-M(K \pi)<0.15$ $\mathrm{GeV}$ candidates

- Background from $D_{s}^{+} \rightarrow \phi\left(\rightarrow K^{+} K^{-}\right) \pi^{+}$suppressed by demanding

- $|M(1,2)-M(\phi)|>8 \mathrm{MeV}$ where 1,2 are any two opposite charge $D^{+}$candidate tracks. 


\section{Systematics}

\section{Systematics}

Systematics are determined by changing the analysis procedure and repeating all calculations.

The following groups or systematic uncertainty were considered:

- The model dependence of the acceptance corrections

- Using the HERWIG MC sample

- Varying the $p_{T}\left(D, \Lambda_{c}\right)$ and $\eta\left(D, \Lambda_{c}\right)$

- Changing the MC fraction of charged $D$ mesons produced in a vector state from 0.6 to 0.5 or 0.7

- The uncertainty of the beauty subtraction

- The $b$-quark $\sigma$ was varied by a factor of 2

- The branching ratios of $b$-quarks to charm hadrons were varied by their uncertainties 


\section{Systematics II}

\section{Systematics II}

- The uncertainty of the tracking simulation

- All momenta varied by $\pm 0.3 \%$ (magnetic field uncertainty)

- Track loss probability varied by $\pm 20 \%$

- Track momentum and angular resolutions varied by ${ }_{-10 \%}^{+20 \%}$ (asymmetric resolution variation arise because MC signals had narrower widths than data)

- The uncertainty of the CAL simulation

- CAL energy scale varied by $\pm 2 \%$

- CAL resolution varied by $\pm 20 \%$

- Efficiencies of CAL first-level trigger varied 


\section{Systematics III}

\section{Systematics III}

- Uncertainties related to signal extraction

- For the $D^{0}$ signals the background parameterisation and fitted range varied

- for the additional $D^{*+}$ signal the area used in background normalisation was varied and the fit was used instead of the subtraction procedure.

- for the $D^{+} D_{s}^{+}$and $\Lambda_{c}^{+}$signals the background parameterisation, fitted region and amounts of mutual reflections were varied

- The uncertainties of the luminosities of the $e^{-} p( \pm 1.8 \%)$ and $e^{+} p( \pm 2.25 \%)$ were included

- The uncertainty in the rate of charm-strange barons 